\title{
Managing uncertainties in LCA dataset selection
}

\author{
Simon Pfeuffer ${ }^{1}$, Daniel Wehner ${ }^{2}$, Raed Bouslama ${ }^{1}$ \\ ${ }^{1}$ University of Stuttgart, Wankelstraße 5, 70563 Stuttgart, DE \\ ${ }^{2}$ Fraunhofer IBP, Wankelstraße 5,70563 Stuttgart, DE
}

\section{Introduction}

There is a requirement for cost effective instruments within the aviation industry to become more sustainable. A well-established methodology to evaluate the sustainability of products and processes is provided by the Life Cycle Assessment (LCA). Currently a trend towards the development of extensive LCA databases on aviation specific materials and processes can be observed. An example for such databases is the Carbon Composites database [1]. The database contains 137 datasets showing inventories with associated environmental impacts of manufacturing carbon fibres and carbon fibre reinforced parts as well as the associated manufacturing processes. Additionally, the technical specifications of the processes responsible for the environmental impacts are reported (e.g. temperatures, holding times etc.). While this information offers great potential for environmental optimization, in early design stages engineers tend to be overwhelmed by the wealth of such information as there is still high uncertainty regarding many environmentally relevant details of their designs, in particular manufacturing related aspects. Consequently, comprehensible means to build a thorough understanding of the LCA information in a database are required to support the awareness about the uncertainties of design decisions. Therefore, we work on answering the following questions:

- How is it possible to cost effectively communicate the uncertainties regarding the environmental impacts of early design decisions?

- How can the process of selecting relevant LCA datasets be supported visually?

\section{Background}

Obtaining a more detailed overview of the content of a database is a challenge within Pleiades. Pleiades is a project within the Clean Sky II program and aims to improve existing eco-design tools by providing a semiautomated workflow to exchange data between a LCA database and a system for assessing ecological risks associated with design decisions. This system 
for Eco-Risk Assessment (ERA) system contains information on materials and processes. In the semi-automated workflow, datasets from the LCA database are mapped to the materials and processes of the ERA system through an interface-tool. One step of mapping the data is currently done manually and consequently time-consuming.

Furthermore, in early design stages uncertainties arise from a missing detailed knowledge of future design decisions. These uncertainties may influence the LCA results [2] and have to be communicated when selecting datasets in order to increase the quality of the data exchange between the ERA system and the LCA database.

\section{Technique}

In order to support the mapping of datasets from a database such as the Carbon Composites database, more advanced techniques have to be considered. The graphical presentation of the datasets and their specific attributes serves as a starting point. A graphical presentation assists the user to understand the different types of uncertainties in early design stages, e.g. when evaluating the ecological impact of various material options. Apart from communicating uncertainties, the graphical presentation makes possible errors resulting from the selection of the LCA dataset visible. The graphical presentations are implemented as a dashboard that provides the user with information about the environmental impacts of the different scenarios represented in the database. The dashboard communicates both, the inherent variability of the datasets in the database and also the environmental improvement potentials of upcoming design decision. As a result, it can further narrow down the design space with regard to material or manufacturing options for example.

\section{Conclusions}

The graphical presentation of the content of the database supports managing the uncertainties in the described design stage by visually communicating the uncertainties in early design decisions. A further development of the technique in form of interactive elements as part of the visualization can make the communication more cost effective. 


\section{References}

[1] Thinkstep AG: Extension database XXII: Carbon Composites. URL http://www.gabi-software.com/support/gabi/gabi-database-2019-lci-

documentation/extension-database-xxii-carbon-composites/, Retrieved April 18, 2019

[2] Heijungs, R., \& Huijbregts, M. A. A review of approaches to treat uncertainty in LCA, Congress on Environmental Modelling and Software. (2004) 